\title{
Phytohormones Ameliorated the Physiological Changes During Post Germination Drought in Maize
}

\author{
Samira Habib ${ }^{1}$ Huma Tariq ${ }^{2}$ Sobia Ashfaq ${ }^{2}$ Naveed Nasir ${ }^{2} \quad$ Syed Abdul Majid ${ }^{3}$ \\ Saira Ishaq ${ }^{4} \quad$ Kiran Fareed ${ }^{5}$ \\ 1, 2 Department of Plant Breeding and Molecular Genetics, University of Poonch, Rawalakot, Azad Jammu and \\ Kashmir , Pakistan \\ 3.Department of Botany, University of Azad Jammu and Kashmir, Muzaffarabad, Pakistan \\ 4.Department of Food Science and Technology, University of Poonch, AJK, Pakistan \\ 5.Department of Horticulture, University of Poonch, AJK, Pakistan
}

\begin{abstract}
Habib et al.,
Novelty statement: Maize is essential cereal crop in Pakistan of rain-fed as well as irrigated lands. During its growing stages it may faces various stresses such as drought that reduced its yield and affects its production. Our experiment revealed that pre-treatment of seeds with gibberellic acid, salicylic acid and sodium nitroprusside are effective to tolerate the effect of drought stress in maize at post germination stage.
\end{abstract}

\begin{abstract}
A pot experiment was conducted to find out effect of gibberellic acid (GA), salicylic acid (SA) and sodium nitroprusside as nitric oxide donor (NO) separately or in combination to mitigate the deviations in physiological parameters evaluated in maize while drought imposed post germination stage. Maize seeds after soaking in $10^{-4}$ $\mathrm{M}$ of above mention chemicals were sown in pots filled with soil. The leaves were sampled at post germination stage. Results indicated that under drought stress, carotenoid and anthocyanin contents increased significantly while chlorophyll $a$ and chlorophyll $b$ remained unchanged at $5 \%$ level of significance. Pre-treatment of solution comprising SA, GA and interaction of GA+SA enhanced the content of these pigments under drought stress. While sodium nitroprusside slightly increased chlorophyll $a$ and $b$ content under drought. Sugar and protein decreased due to drought stress and sugar enhanced due to effect of NO only but protein content did not increase by any one of applying hormones under drought stress. Likewise GA and SA enhanced accumulation of proline which acts as osmolyte and scavenger of free radical to defend cell injury against drought stress. Similarly activities of oxidative enzymes such as peroxidase and polyphenol oxidase increased under drought stress and responded differentially by GA, SA etc, while ascorbate peroxidase and catalase activities decreased due to drought stress which became increased by effect of NO. Results revealed that pretreatment of seeds with GA, SA, $\mathrm{GA}+\mathrm{SA}$ and $\mathrm{NO}$ are effective to tolerate the effect of drought stress in maize at post germination stage.
\end{abstract}

Keywords: Drought, Enzymes, Maize, osmolyte, Pigments

DOI: $10.7176 / \mathrm{JBAH} / 11-15-01$

Publication date:August $31^{\text {st }} 2021$

\section{Introduction}

Maize is third most essential cereal crop after wheat and rice. Pakistan maize is main kharif crop of rain-fed as well as irrigated lands. During maize growth period with high possibility of drought more than $35 \%$ of crop is planted under rain fed conditions. Abiotic stress affects yield of maize crop and may cause yield reduction more than $50 \%$. Drought affects every feature of plants growth and responsible to restrictive production of crop. By accumulating cyto-compatible osmolytes including proline, betaines and polyols, plants tend to adapt drought (Rao et al., 2013). Drought affects quickly the processes associated to cell turgidity mainly the growth of the meristems. Under drought condition, increase in abscisic acid (ABA) contents and decrease in gibberellic acid (GA) and cytokinin (CK) suggested that due to alteration in hormonal equilibrium, the reduction of growth might be resulted (Akter et al., 2014). To cope with drought stress, therefore, exogenous application of hormones might be a suitable approach (Akter et al., 2014). The yield of several crops may increase due to decrease in stress made inhibition of growth, leaf area, improved photosynthetic rates and plant dry matter production by treatment of seed and foliar applications of chemicals such as glycinebetaine, kinetin and salicylic acid. Salicylic acid naturally occurring phytohormone, play a role as a significant signaling molecule enhances tolerance under abiotic stresses. Salicylic acid plays a vigorous role in ion transport and uptake and plant growth. It also involved to endogenous signaling of plant for activate defence mechanism against pathogens. Photosynthesis, plants water relation, stomatal regulation and growth under abiotic stress conditions can also significantly manage by salicylic acid (Rao et al., 2012). Gibberellins are a family of compounds generally have growth-promoting function. Drought stress resistance in maize increased by exogenous application of GA3 that improved chlorophyll content, maintained membrane permeability, leaf relative water content and macro nutrient content in leaves (Kaya et al., 
2006). The Nitric oxide in plant cells is suggested to be one of the important second messengers. The nitric oxide synthase (NOS) synthesized most of NO in animal systems. Though, the NOS has not until now remote in plants but NOS activity for NO production can be noticed. The proteins like NOS probably localized in maize cytoplasm suggested by immunological analysis. Several physiological processes to abiotic stresses can also mediate by exogenous application of NO. The sodium nitroprusside NO donor application confers resistance in drought (Sang et al., 2008) stress. Exogenous application of SNP under drought stress prevented drought induced decreased relative water content membrane stability and growth performance, by improving proline accumulation and stimulation of oxidative enzymes cause a decreased lipid peroxidation and $\mathrm{H}_{2} \mathrm{O}_{2}$ content (Nasibi and Kalantari, 2009). Phytohormones have challenging and continues one of the greatest active areas of plant biology research because of their pleiotropic effect, unraveling the functions.

The objective of this project was to study the role of phytohormones like gibberellic acid, salicylic acid and sodium nitroprusside as nitric-oxide donor separately or in combination to mitigate the changes in physiological parameters developed by controlling the application of irrigating water during post germination stage.

\section{Material and Methods}

The experiment was conducted in triplicates. The seeds were soaked in $10^{-4}$ molar solution of gibberellic acid, salicylic acid, salicylic acid + gibberellic acid, sodium nitroprusside as nitric oxide donor and distilled water as control for four hours. After treatment seeds were grown in pots having soil, making two sets of each. Two group of seedlings control and drought stress were examined.Seedlings were harvested when attained six to seven leaf stage according to plain and stored in polythene bags, placed in refrigerators at $4^{\circ} \mathrm{C}$.

\section{Plant Pigments}

The amount of photosynthetic pigments were recorded by method of Lichtenthaler (1987). The chlorophyll $a$, chlorophyll $b$, carotenoid and anthocyanin were calculated using the equation (Sims and Gamon 2002).

Protein

Soluble protein content in fresh leaves was measured by the process described by Bradford (Bradford, 1976).

Sugar

Sugar content of samples were assessed according to Dubois et al. (1956) method.

Proline

Proline determined by method of Bates et al. (1973).

Catalase

sample was homogenized in $10 \mathrm{ml}$ of 0.2 mole phosphate buffer $(\mathrm{pH} 7.0)$ in pre cooled mortar and pestle at $4^{\circ} \mathrm{C}$ and centrifuged at $8000 \mathrm{rpm}$ for 20 minutes. The catalase activity assayed according to method of Aebi (1984).

\section{Peroxidase}

One gram leaf sample was homogenized in $10 \mathrm{ml}$ of 0.2 mole phosphate buffer (pH 7.0) in pre cooled mortar and pestle at $4^{\circ} \mathrm{C}$ and for 20 mint centrifuged at $8000 \mathrm{rpm}$. Supernatant was used for peroxidase analyze according to the procedure used by Tutschek (1979).

\section{Ascorbate-peroxidase}

The supernatant obtained for peroxidase assay was used to measure ascorbate peroxidase enzyme activity (Bartoli et al., 1999).

Polyphenol Oxidase

The polyphenol oxidase activity was measured according to Shinshi and Noguchi (1975) by using supernatant obtained for peroxidase activity.

\section{Statistical analysis}

Collected data subjected to ANOVA and mean value at 5\% level of significance was verified by Duncan's Multiple Range Test utilizing computer program MSTAT-C.

\section{Results and Discussion}

\subsection{Results:}

\section{Pigment Content}

Chlorophyll contents are the indicators of photosynthetic activity of the plants. Many factors naturally affect the contents and ultimately influenced the photosynthesis. Plant growth regulators play role in mitigating the changed behavior of the physiological parameters. In present study, drought induced changes in chlorophyll contents were evaluated and found differential affects. Chlorophyll $a$ contents remained unchanged under drought stress imposed at post germination stage. Research work in two lines of okra show same result where chlorophyll a remain unaffected under drought stress (Jaleel et al., 2009). Due to the effect of gibberellic acid, salicylic acid, salicylic acid+ gibberellic acid and nitric oxide chlorophyll contents increased, although non-significant. The effect of gibberellic acid and salicylic acid+ gibberellic acid on chlorophyll $a$ contents was observed unchanged under drought (Figure 1). Salicylic acid decreased the content under drought stress. Only the nitric oxide 
responded well and an increased chlorophyll $a$ contents under drought stress were noticed. At post germination gibberellic acid and salicylic acid did not responded in maintaining the drought stress due to their activities in other physiological involvement showing no role at the stage of post germination.

The main function of chlorophyll $b$ is to collect light energy, functioning together with carotenoids and chlorophyll $a$. In present study chlorophyll $b$ content behave differentially as compared to chlorophyll $a$ under drought induced stress. Content of chlorophyll $b$ increased under drought stress from 3.95 to 4.05 nonsignificantly. Increased in chlorophyll $b$ contents, a drought stress adaptation in order to rise photosynthetic capability (Figure 2). Same result of research in two lines of okra indicate that increase in chlorophyll b content caused by drought stress (Jaleel et al., 2009). Gibberellic acid decreased the content to some extent without drought but in drought cause still increased the content. Salicylic acid cause to decrease the content under drought stress which increased due to stress. While combined effect of gibberellic acid + salicylic acid increased the content without drought and to some extent decreased under drought. Similarly nitric oxide cause to decrease the content without drought and under drought content slightly decreased which increased due to stress. Our findings indicated that combined effect of salicylic acid + gibberellic acid and nitric oxide mitigate the drought stress effects.

Carotenoids have crucial functions in photo protection and photosynthesis. They have important role in stabilizing membranes and inhibit lipid peroxidation by antioxidant activity. In present study, carotenoids content increased significantly under drought stress imposed at post germination stage. Pretreatment of gibberellic acid + salicylic acid and gibberellic acid maximum enhance the carotenoids content under drought induce as well as without drought state (Figure 3). Salicylic acid also show to increase the content under drought induce state. Nitric oxide show to increase the content without but not to cause increased much under drought induce state. It showed that combination of gibberellic acid + salicylic acid and separately both helpful for plant to tolerate drought stress. Carotenoids antioxidant mechanisms are distributed into four categories, they are singlet oxygen quenching, sun screening, radical scavenging and excessive light energy releasing through xanthophyll cycle. They can also prevent photosensitized oxidation, thus they can act as competent quencher of singlet oxygen (Praveena and Murthy, 2014).

Anthocyanins are coloured pigments that provide flowers their distinctive purple, red and blue shades. Anthocyanin accumulation in leaves is connected with ontogenetic, abiotic and biotic effects. Anthocyanin can be responsible a photoprotective role, in the perspective of evidence for its role as an antioxidant, its distribution in leaves and its synthesis in seedlings. In current study, anthocyanins content increased significantly under drought stress show that plant resist the effect of drought which further enhanced by pre-treatments of gibberellic acid, salicylic acid and combined effect of both gibberellic acid + salicylic acid. Accumulation of anthocyanin under drought stress suggest that the play a photoprotective role because of their capability to absorb light between 400 to $600 \mathrm{~nm}$, they protect chloroplast from excess irradiance. In our study accumulation of anthocyanin and carotenoid increase under drought stress suggest that under drought stress they are intricate in photoprotection (Figure 4), which is an agreement with earlier investigation (Merzlyak et al. 2008; Gould et al., 200; Close and Beadle 2003; Hoch et al., 2001).

Important role of sugars in plant metabolism as a product of hydrolytic process and energy production. Sugar provide resistance against drought stress help the cell to maintained structural integrity of membrane and their dehydrated state. Our results indicate that sugar content decreased significantly in maize plant at post germination stage due to drought stress (Figure 5). Pre-soaking treatment with NO is effective to increase sugar content in drought state as compared to non-treated plant to increase cell osmotic adjustment. Which suggest that NO donor sodium nitro-prusside effect the gene expression of somatically functional molecule as stated by Shehab et al. (2015) observed increase in concentration of sucrose by NO under drought stress rice plants. Therefore sucrose appears to show a critical function in the assimilation of plant development and in osmotic adjustment and NO contribute in this regard to increase sugar content in our investigated results.

Proline accumulates in many plants in response to drought stress. It can act as signalling molecule to moderate mitochondrial function and activate specific gene expression necessary for plant rescue from stress. In present study interaction between hormones and drought observed that maximum proline was found in maize plant at post germination stage treated with gibberellic acid followed by salicylic acid under drought. This showed that proline content increase by effect of gibberellic acid and salicylic acid in drought as compared to non-treated plant (Figure 6). Pre-treatment with salicylic acid adds to accumulation of proline under stress by maintaining a higher level of ABA in wheat seedlings (Sakhabutdinova et al., 2003).

Abiotic stresses usually cause protein dysfunction. In present study drought stress result in significantly decreased in concentration of protein in maize plant as compared to control as revealed in (Figure 7). Our result showed that protein content increase in maize plant due to soaking treatment of different hormones as compared to non-treated plant, without drought condition. Maximum content was observed due to effect of salicylic acid and after that nitric oxide in non-drought state. But soaking treatment did not effective to increase the protein content in drought. Water stress cause to decrease in the protein content of tissue by inhibit the incorporation of 
amino acids into proteins. Water deficit inhibits protein synthesis at the ribosomal level (Sobhkhizi et al., 2014). Reduction in the protein content would be characteristic sign of oxidative stress that in drought stressed plants has been observed frequently (Moran et al., 1994; See et al., 1992). Decreased in protein content was also recorded by Kala and Godra (2011) in moisture stress Ziziphus mauritiana.

\section{Oxidative Enzymes}

Under environmental stresses ROS produced in plants which can be detoxify by oxidative enzymes like catalase, peroxidase, ascorbate-peroxidase and polyphenol oxidase.

Catalase (CAT) is one of the main antioxidant enzyme which degrades hydrogen peroxide into oxygen and water. In stresses full environment such as drought $\mathrm{H}_{2} \mathrm{O}_{2}$ produced in the peroxisome by photorespiration in such conditions CAT enzyme activities are important to tolerate stresses for plants. In our investigated data catalase activity decreased due to induced drought stress in maize at post germination stage. But pre-treatment of seeds by different hormones was effective to increased catalase activity under drought induced stress. Maximum activity was recorded by effect of nitric oxide (NO) under drought stress followed by salicylic acid + gibberellic (Figure 8). Similarly salicylic acid and gibberellic acid separately also helpful to increase the catalase activity under drought stress as compared to non-treated plant. Catalase is one of the chief antioxidant enzymes that degrades $\mathrm{H}_{2} \mathrm{O}_{2}$ into oxygen and water (Scandalios et al., 1997). Pre-treatment of nitric oxide remarkably increased this depress character. Under stress conditions, nitric oxide seems to help as an anti-oxidant agent that able to scavenge hydrogen peroxide to protect plant cells from oxidative damage were in agreement with Sang et al., 2008: Nasibi and Kalantari, 2009.

Peroxidase also defends the cells against the damaging effect of $\mathrm{H}_{2} \mathrm{O}_{2}$ by catalysing its disintegration through oxidation of phenolic and endiolic co substrates. In present study, peroxidase activity naturally increased under drought stress to fight against the damaging effect of drought in maize to tolerate stress. Lum et al. (2014) also reported increase in peroxidase activity under drought stress in upland rice drought tolerant verities. Pretreatment of gibberellic acid cause to decrease the enzyme activity without drought but still increased the activity in drought (Figure 9). Salicylic acid also decrease the enzyme activity in normal condition and to some extent in drought. Combined effect of gibberellic acid + salicylic acid sow to increase the enzyme activity in in normal condition and in drought. While nitric oxide cause to increase the enzyme activity without drought but decrease to some extent in drought show that it might take part to release plant from drought stress.

Ascorbate-peroxidase is an enzyme that detoxifies $\mathrm{H}_{2} \mathrm{O}_{2}$ using ascorbate as a substrate. They catalyse reaction that transfer electrons from ascorbate to a peroxide, producing water and de-hydroascorbate as products. Our result indicate that pre-soaking treatment of seeds with sodium nitroprusside as nitric oxide donor was effective to increase the ascorbate-peroxidase activity under drought stress which become decrease in non-treated plant due to drought stress (Figure 10). Hence sodium nitroprusside as nitric oxide donor reduce the damaging effect drought stress by increasing the activity of ascorbate-peroxidase. Using ascorbate as substrate, ascorbate peroxidase activity break down hydrogen peroxide. Though, some scientists have related declines in this enzyme at water deficient stage. Zhang and Kirkham (1996) found sorghum plants cultivated under drought stress decreasing in ascorbate-peroxidase and stated that ascorbate without ascorbate peroxidase take part in the removal of hydrogen peroxide.

Polyphenol oxidases (PPOs) ubiquitous copper-containing enzymes. Which used molecular oxygen to oxidize ortho-diphenolic compounds including catechol and caffeic acid and to their respective quinones. PPOsgenerated quinones are extremely reactive and may cross-link or alkylate proteins that leading to the usually detected brown pigments in injured plant tissues (Mayer 2006). In present study polyphenol activity increased under drought induced stress at post germination stage in maize and enhance by effect of salicylic acid and nitric oxide (Figure 11). These results indicate that by synthesizing more amount of PPO, tolerates drought stress, in agreement with Shinde and Laware (2015). While gibberellic acid and combined effect of gibberellic acid + salicylic acid decrease the PPO activity which increased under drought stress show that they mitigate the drought stress and release the plant stress effects.

\section{Discussion}

Drought is most severe problem effecting plant growth and development. Hence in our results seed soaking treatment with different hormones was most effective to reduce the damage caused by drought. Drought stress not only lead to damage photosynthetic pigments but also cause thylakoid membranes deterioration. Therefore, decrease in photosynthetic capability expected when plants exposed to drought stress (Mafakheri et al., 2010). The reduction in chlorophyll contents commonly observed under drought stress. Effect of drought on photosynthetic pigments show both significant and non-significant results. Our data indicate that drought stress did not affect the content of chlorophyll $a$ significantly statically in maize plant. Kulshrehtha et al. (1987) also found in wheat no significant effect of water stress on chlorophyll content. While chlorophyll $b$ content increased under drought imposed stress. Present study show to increase total chlorophyll content under drought stress as revealed 
by graph-11 and enhanced by NO. There are also some reports, show greater accumulation of chlorophyll under water stress condition (Estill et al., 1991, Pirzad et al., 2011). Increase chlorophyll content in some cultivars of blackgram (Vigna mungo) and reduction in others under water drought stress also reported by Ashraf and Karim (1991) and proposed that it might be due to deviation in chlorophyll synthesis amongst cultivars mediated by the variations in activities of definite enzymes that intricate in biosynthesis of chlorophyll.

Total chlorophyll under drought stress increased as compared to control in present study.

Present study show that chlorophyll $\mathrm{a} / \mathrm{b}$ ratio decreased under drought stress significantly in maize at post germination stage (Figure 13). Increase the content of chlorophyll b, where chlorophyll $a$ remain unaffected cause to decrease in chlorophyll $a / b$ ratio under drought stress also reported in two lines of okra (Jaleel et al., 2009). Soaking treatment of seeds by growth regulator show different pattern under drought and non-drought conditions. Gibberellic acid cause to increase significantly chlorophyll a/b ratio without drought but did not increased the depressed ratio in drought. Salicylic acid and combined effect of salicylic acid+ gibberellic acid increased the chlorophyll a/b ratio under drought and non- drought condition. Rao et al. (2012) also show that foliar application of salicylic acid cause to increase the chlorophyll content. Maximum increase of ratio due to effect of nitric oxide both in drought and normal condition Which indicated that nitric oxide best hormone to increase the chlorophyll content and enhance the photosynthesis process. Alvi et al. (2014) also reported that SA and NO pre- treatment decreased the harmful effects of water stress by increasing antioxidant activities that cause to increase shoot fresh weight, chlorophyll content and membrane stability index and decreased the level of MDA and LOX activities.

While in present study content of anthocyanin and carotenoid were significantly increased under drought stress and by soaking treatment of hormones. Best hormones to increase the carotenoid content were GA+SA, GA, SA and NO according to our investigated data. Carotenoids have crucial functions in photo protection and photosynthesis. They have important role in stabilizing membranes and inhabit lipid peroxidation by antioxidant activity as stated Demmig and Adams (1992), Frank and Cogdell (1996) and Niyogi (1999). The carotenoids also have essential role in the radiation less dissipation of excess energy and assembly of light harvesting complex as reported by Streb et al., (1998); Munne-Bosch and Alegre (2000). Increase in concentration of anthocyanin was reported in our result under drought stress as compared to non-drought maize plant. Also treatment of different hormones enhance anthocyanin content such as SA, GA and GA+SA suggest that these hormones are effective to adapt the plant to fight against deleterious effect of drought stress. Accumulation of anthocyanin under drought stress suggest that the play a photoprotective role because of their capability to absorb light amid 400 to $600 \mathrm{~nm}$, they protect chloroplast from excess irradiance. In our data accumulation of anthocyanin and carotenoid increase under drought stress suggest that under drought stress they are intricate in photoprotection, which is an agreement with earlier investigation (Merzlyak et al. 2008; Gould et al., 200; Close and Beadle 2003; Hoch et al., 2001).

Another important compound proline may be affected by drought stress. In usual cases concentration of these molecular compound increase in response to water deficient stress by stress resistance verities. Proline and sugar act as osmoprotectant during drought stress. Our data revealed that concentration of sugar decreased under drought stress for the reason that anaerobic respiration start enlarged under great stress. Plants for their survival preferred to convert other product to activate anaerobic respiration. Besides in drought stress, stomata have not fully closed and uptake of carbon dioxide to aerobic respiration. Our results were in agreement with Haeo et al., 2015. While soaking treatment of grain with NO was effective to increase the concentration of sugar (1.670) in stressed plant as compared to non-treated plant (1.393) as indicated in our investigated data. Which suggest that NO donor sodium nitro- prusside effect the gene expression of somatically functional molecule as stated by Shehab et al. (2015) observed increase in concentration of sucrose by NO under drought stress rice plants. Therefore sucrose appears to show a critical function in the assimilation of plant development and in osmotic adjustment and NO contribute in this regard to increase sugar content in our investigated results. Sugar as osmoprotectors stabilize membranes and proteins by substituting water in making hydrogen bonding with phospholipid phosphate groups and polypeptide polar residues, reviewed by Shehab et al. (2010). In our investigated data mean values of proline contents in drought and non- drought state were non-significant. But effect of different hormones on proline content show significant difference. Interaction between hormones in drought and non-drought stress also indicate maximum content was due to effect of GA (0.306) and SA (0.273). Which show that GA and SA enhance proline accumulation for drought tolerance propose. Proline play vital role in cellular osmotic adjustment and scavenge detoxify oxidants also reported by Ashraf and Foolad (2007); Seki et al. (2007); Ali et al. (2008). Rao et al. (2012) recommend that foliar application of SA in maize can help to decrease the drought stress effects. Drought usually cause protein dysfunction. According to our investigated data protein content decrease significantly under drought stress. Maximum protein content (3.700) without drought decrease to (1.842) under drought. Which proved that drought adversely affect protein content of maize plant. While hormonal treatment increase the protein content in normal irrigated plant but no one hormone increase the content under drought stress. Water stress inhabit the incorporation of amino acid into protein. 
Drought stress cause change in protein synthesizing apparatus and cause a considerable decreased in protein synthesizing capacity. Decrease in protein contents under drought stress were also recorded by other scientist (Sobhkhizi et al 2014; Kala and Godra 2001; Moran et al., 1994; Genkel et al., 1967; Hsiao et al., 1970).

Drought cause oxidative stress which responsible for increased production of ROS comprising singlet oxygen, superoxide, hydroxyl radical and hydrogen peroxide. These ROS can attack proteins, lipids, nucleic acid and carbohydrates. Several enzymes detoxify ROS including Catalase, peroxidase and ascorbate peroxidase and polyphenol oxidase. Previous study data have proved that higher activities of antioxidant enzymes might be involve to improved tolerance of drought by enhancing protection capability against oxidative harm. Our investigated data indicate that oxidative enzymes such as peroxidase and polyphenol oxidase activity increased under drought stress and enhanced by hormonal treatment for better stress tolerance. In our investigated data peroxidase activity increased under drought stress (5.507) as compared to non-drought (4.435). By application of GA peroxidase activity increased (5.844) in drought and by NO (6.400). Rise in POX activity serving to overcome harm to tissue metabolism against oxidative stress by decreasing hydrogen peroxide toxic levels formed in cell metabolism. Our results were in agreement with previous studies (Sudhakar et al., 2001 and Hojati et al., 2011). Catalase activity decreased significantly under drought stress. Soaking treatment with NO show maximum catalase activity (9.300) followed by GA (8.400) and SA+GA (8.200) as compared to non-treated plant (6.300). Which indicate that NO, GA and interaction of GA+SA take part to detoxify the effect of ROS by stimulating the catalase activity. By photorespiration in peroxisomes $\mathrm{H}_{2} \mathrm{O}_{2}$ produce which is degrade by catalase. (Noctor et al., 2000). Catalase is one of the chief antioxidant enzyme that degrades $\mathrm{H}_{2} \mathrm{O}_{2}$ into oxygen and water (Scandalios et al., 1997). In present study polyphenol activity increased under drought induced stress at post germination stage in maize and enhance by effect of salicylic acid and nitric oxide. These results indicate that by synthesizing more amount of PPO, tolerates drought stress, in agreement with Shinde and Laware (2015). While gibberellic acid and combined effect of gibberellic acid + salicylic acid decrease the PPO activity which increased under drought stress show that they mitigate the drought stress and release the plant stress effects. There were numerous studies that supported under stress condition NO induced stimulations of main oxidative enzymes like CAT, APX and SOD reported by Sang et al. (2008); Tian and Lei (2007).

\section{Conclusion}

It's well known that drought adversely affect plant growth and yield. Plants adopt different defence mechanisms for their survival. Our study revealed that under drought stress defensive pigments carotenoids and anthocyanins increased to defend photosynthetic machinery from oxidative damage by acting as antioxidants. And pretreatment of hormones comprising SA, GA and interaction of GA+SA enhance the content of these pigments under drought stressed. While content of chlorophyll $a$ remained unchanged but slightly increased due to effect of sodium nitroprusside as NO donor under drought stress. Stress markers sugar and proline are osmoprotectant . Sugar content decreased under drought stress but pre- treatment of sodium nitroprusside increase the content to maintain cell turgidity. Similarly GA and SA enhance accumulation of proline which acts as osmolyte and scavenger to defend cell against stress. While protein content decreased due to drought stress and the treatment of any hormones could not be effective to increase the content under stress. Drought stress caused to production of ROS, which attack lipids proteins and carbohydrates. Oxidative enzymes activities detoxify the damage of ROS as a result peroxidase and polyphenol activities increased under drought stress and behave deferentially by the pre-treatment of GA, SA and NO. But catalase and ascorbate-peroxidase activity decrease under drought stress which have been increased due to effect of NO. Hence it is suggested that soaking treatment of seeds by SA, GA and NO might be effective to reduce the damaging effect of drought at post germination stage of maize plant in different agro-ecological zones.

\section{Acknowledgement}

All authors are great full to Department of Botany, University of Azad Jammu and Kashmir, and Muzaffarabad, Pakistan for allowing lab experiment for this study.

\section{Conflict of Interest}

There is no conflict of interest.

\section{Literature Cited}

Aebi, H. (1984). Catalase in vitro. Method in enzymology. Florida: Ascad. Press, 105: 114-121.

Akter, N., M. R. Islam, M. A. Karim and T. Hossain.2014. Alleviation of Drought Stress in Maize by Exogenous Application of Gibberellic Acid and Cytokinin. Journal of crop science and Biotechnology, 17 (1): 41-48.

Ali, Q., M. Ashraf, M. Shahbaz and H. Humera. (2008). Ameliorating effect of foliar applied proline on nutrient uptake in water stressed maize (Zea mays L.) plants. Pakistan journal of botany, 40: 211-219.

Ashraf, M. and F. Karim. (1991). Screening of some cultivars/lines of black gram (Vigna mungo I., Hepper) for 
resistance to water stress. Tropical Agriculture, 68: 57-62, 1991.

Ashraf, M. and M. R. Foolad. (2007). Roles of glycine betaine and proline in improving plant abiotic stress resistance. Environment and Experimental. Botany, 59: 206-216.

Bartoli, C. G., M. Simontacchi, E. Tambussi, J. Beltrano, E. Montaldi and S. Puntarulo. (1999). Drought and watering-dependent axidative stress: effect on anti-oxidant content in Triticum aestivum L. leaves. Journal of Experimental Botany, 50: 375-383.

Bates, L. S., R. P. Waldern and I. D. Teare, (1973). Rapid of adaptation to cyclic water stress in two south determination of free proline for water-stress Australian bread wheat cultivars. Plant Soil, 39: 205-208.

Close, D. C. and L. C. Beadle. (2003). The Ecophysiology of Foliar Anthocyanin. The Botany Review, 69 (2): $149-161$.

Dubois, M., K. A. Gilles and J. D. Hamilton. (1956). Colorimetric method for determination of sugars and related substances. Analytical Chemistry, 28: 350-35.

Estill, K., R. H. Delaney, W. K. Smith and R. L. Ditterline. (1991). Water relations and productivity of alfalfa leaf chlorophyll variants. Crop Sci, 31: 1229-1233.

Genkel, P. A., N. A. Satarova and E. K. Tvorus. (1967). Effect of Drought on Protein Synthesis and the State of Ribosomed in Plants. Fiziol, Rast, 14: 898-907.

Hojati, M., S. Sanavy, M. Kareimi, and F. Ghannati. (2011). Responses of growth and antioxidant systems in Carthamustinctoris L. under water deficit stress. Acta Phsiol Plant, 33:105- 112.

Hsiao, T.C. (1970). Rapid Changes in the Levels of Polyribosomes in Zea mays in Reponse to Water Stress. Plant Physiology, 46 (2): 281-285.

Jaleel, C.A., P. Manivannan, A. Wahid, M. Farooq, H. J. Al-juburi, R. Somasundaram and R.

P. Vam. (2009). Drought stress in plants: a review on morphological characteristics and pigments composition. International Journal of Agriculture and Biology, 11: 100-105.

Kala, S. and A. K. Godara. (2011). Effect of moisture stress on leaf total proteins, proline and free amino acid content in commercial cultivars of Ziziphus mauritiana. Journal of Scientific Research, 55: 65-69.

Kaya, C., A. L. Tuna, A. C. Alfredo and Alves. (2006). Gibberellic acid improves water deficit tolerance in maize plants. Acta Physiol. Plant, 28(4): 331-337.

Kulshrehtha, S., D. P. Mishra, R. K. Gupta. (1987). Changes in contents of chlorophyll, proteins and lipids in whole chloroplasts and chloroplast membrane fractions at different water potential in drought resistant and sensitive genotypes of wheat . Photosynthetica, 21: 65- 70.

Lei, Y. C. Yin and C. Li. (2007). Adaptive responses of Opulus przewalskii to drought stress and SNP application. Act. Physiol. Plant, 29: 519-526.

Lichtenthaler, H. K. (1987). Chlorophylls and carteniods: pigments of photosynthetic biomembranes. Methods Enzymology, 148: 350-382.

Lum, M.S., M. M. Hanafi, Y. M. Rafii, and A. S. N. Akmar. (2014). Effect of drought stress on growth, proline and antioxidant enzyme activities of upland rice. Journal Animal and Plant Science, 24(5): 1487-1493.

Mafakheri, A., A. Siosemardeh, B. Bahramnejad. (2010). Effect of drought stress on yield, proline and chlorophyll contents in three chickpea cultivars. Australian Journal of Crop Science 4: 580- 585, 2010.

Mayer, A. M. (2006). Polyphenol oxidases in plants and fungi: going places? A review. Phytochemistry, 67: 2318-2331.

Moran, J. F., M. Becana, I. Iturbe-Ormaetxe, S. Frechilla, R. V. Klucas, P. Aparicio-Tejo. (1994). Drought induces oxidative stress in pea plants. Planta, 194: 346-352.

Nasibi, F. and K. M. Kalantari. (2009). Influence of nitric oxide in protection of tomato seedling against oxidative stress induced by osmotic stress. Acta Physiol Plant, 31: 1037-1044.

Noctor, G., S. Veljovic-Jovanovic and C. H. Foyer. (2000). Peroxide processing in photosynthesis: antioxidant coupling and redox signalling. Philosophical Transactions of the Royal Society of London. 355: 1465-1475.

Pirzad, A., M. R. Shakiba, and S. Zehtab-Salmasi. (2011). Effect of water stress on leaf relative water content, chlorophyll, proline and soluble carbohydrates in Matricaria chamomilla L. Journal of Medicine\& Plants Research, 5: 2483-2488.

Praveena, B. and S. D. S. Murthy. 2014. Role of photosynthetic pigments in protection against oxidative damage. I. J. P. A. E. S. 4(1): 2231-4490.

Rao, S. R., A. Qayyum, A. Razzaq, M. Ahmad, I. Mahmood and A. Sher. (2012). Role of foliar application of salicylic acid and L-tryptophan in drought tolerance of maize. Journal of agriculture and plant science, 22(3): 768-772.

Sakhabutdinova, A.R., D. R. Fatkhutdinova, M. V. Bezrukova and F. M. Shakirova. (2003). Salicylic acid prevents the damaging action of stress factors on wheat plants. Journal of Plant physiology, special issue: 314-319.

Sang J., M. Jiang, F. Lin, S. Xu, A. Zhang and M. Tan. (2008). Nitric oxide reduced hydrogen peroxide accumulation involved in water stress induced subcellular antioxidant defense in maize plants. Journal of 
agriculture and plant science, 50: 231-243.

Scandalios J. G., L. M. Guan and A. Polidoros. (1997). in Oxidative Stress and the Molecular Biology of Antioxidant Defenses. Scandalios JG (ed.) Cold Spring Harbor Lab. Press, Plainview, NY) 343-406.

Shaddad, M. A. K., M. H. Abd El-Samad and H. T. Mohammed. (2011). Interactive Effects of Drought Stress and Phytohormones or Polyamines on Growth and Yield of Two Maize (Zea maize L.) Genotypes. Journal of Plant Science, 2: 790-807.

Shehab, G., K. A. Osama and H. S. El-beltagi. (2010). Effects of Various Chemical Agents for Alleviation of Drought Stress in Rice Plants (Oryza sativa L.). Not. Bot. Hort. Agrobot. Cluj, 38 (1): 139-148.

Shinde, B. M. and S. L Blaware. (2015). Investigation of water stress on antioxidant enzyme activities in groundnut varieties (Arachis hypogaea L). International Journal of Agriculture Biological Research, 5(1): 29-33.

Sims, D. A. and J. A. Gamon. (2002). Relationship between leaf pigment content and spectral reflectance across a wide range of species, leaf structure and developmental stages. Remote Sensing Environment, 81: 337354.

Sudhakar, C., A. Lakshmi and S. Giridarakumar. (2001). Changes in the antioxidant enzyme efficiacy in two high yielding genotypes of mulberry (Morus alba L.) under NaCl salinity. Plant Science, 161: 613-619.

Tian, X. and Y. Lei. (2007). Physiological responses of wheat seedlings to drought and UV-B radiation. Effect of exogenous sodium nitroprusside application. Russian Journal of Plant Physiology, 54: 676-682.

Tutschek, R.(1979). Characterization of peroxidase Sphagnum megallanicum. Phytochemistry, 18: 1437-1439.

Zhang, J., M. B. Kirkham. (1996). Antioxidant responses to drought in sunflower and sorghum seedlings. New Phytologist, 132: 361-373.

Figure-1. Effect of growth hormones on chlorophyll contents $(\mathrm{mg} / \mathrm{g})$ of drought stressed maize plants.

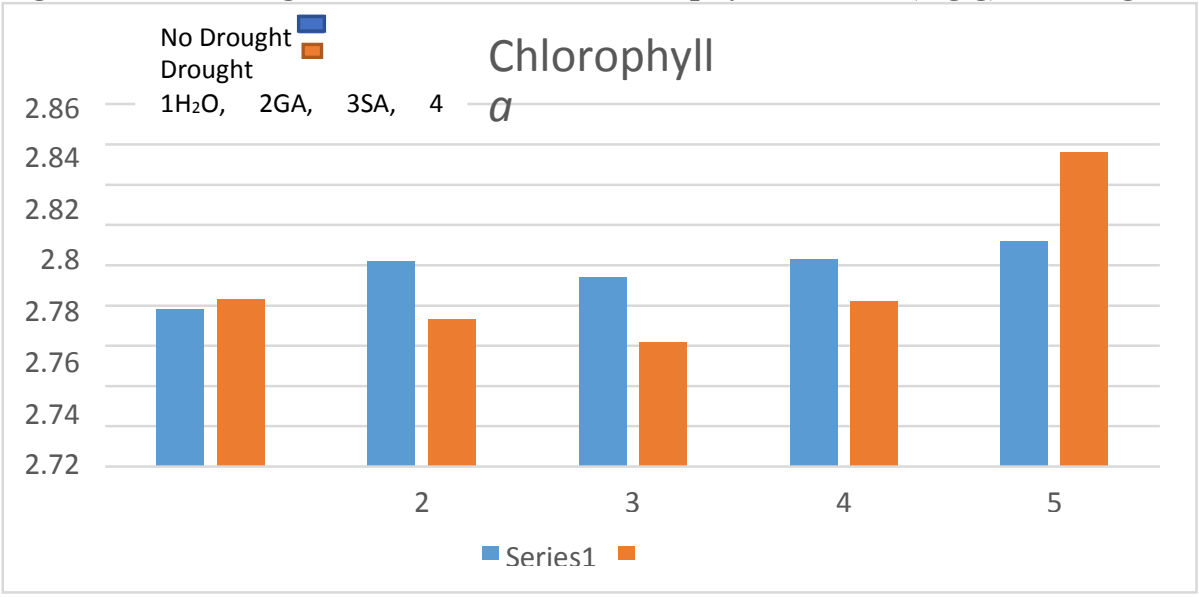

Figure-2. Effect of different growth hormones on chlorophyll $b$ content $(\mathrm{mg} / \mathrm{g}$ fw) of post germination drought stress maize.

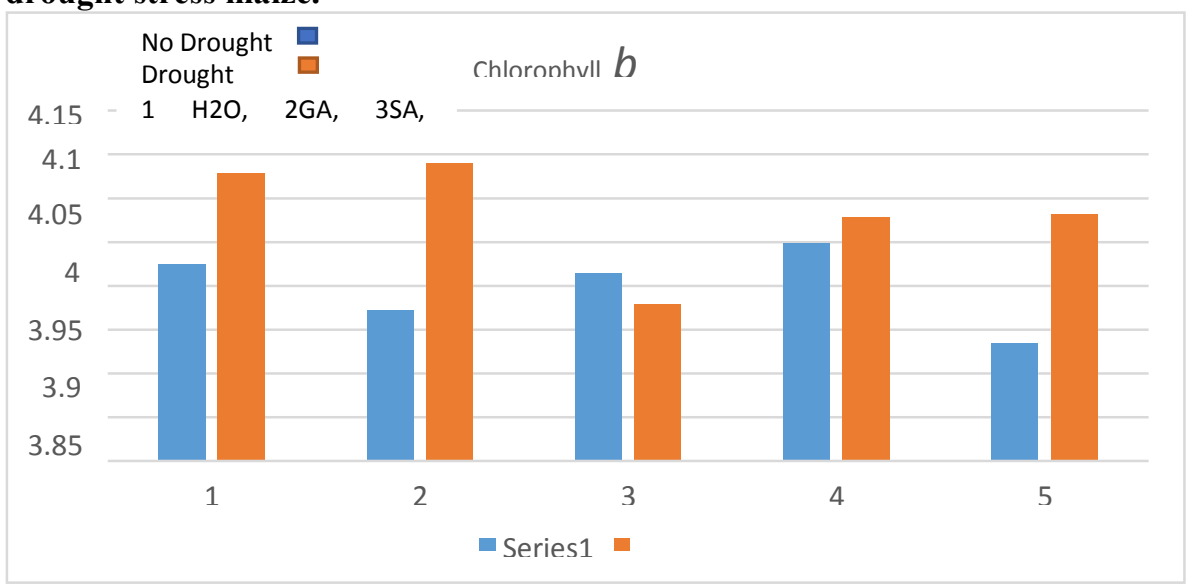


Figure-3 Effect of different hormones on carotenoid pigment (mg/g fw) of post germination drought stressed maize plant.

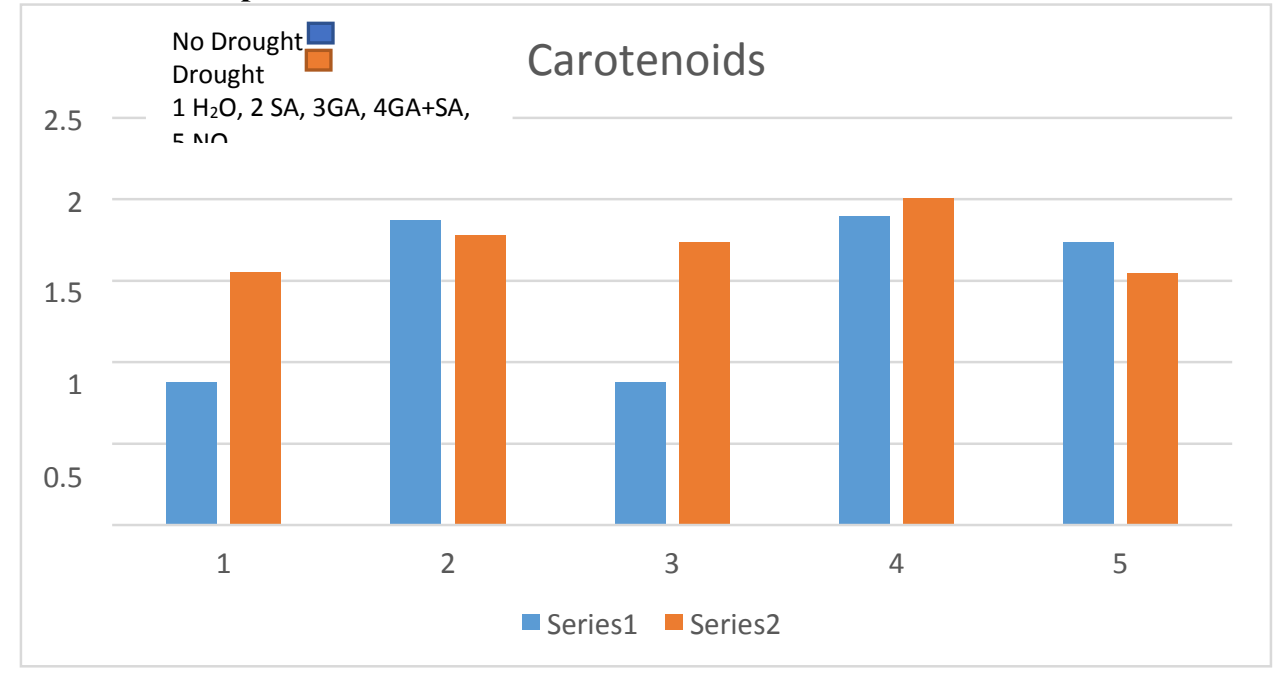

Figure-4 Effect of different hormones on anthocyanin $(\mathrm{mg} / \mathrm{g} \mathrm{fw})$ of maize under drought stress during post germination stage.

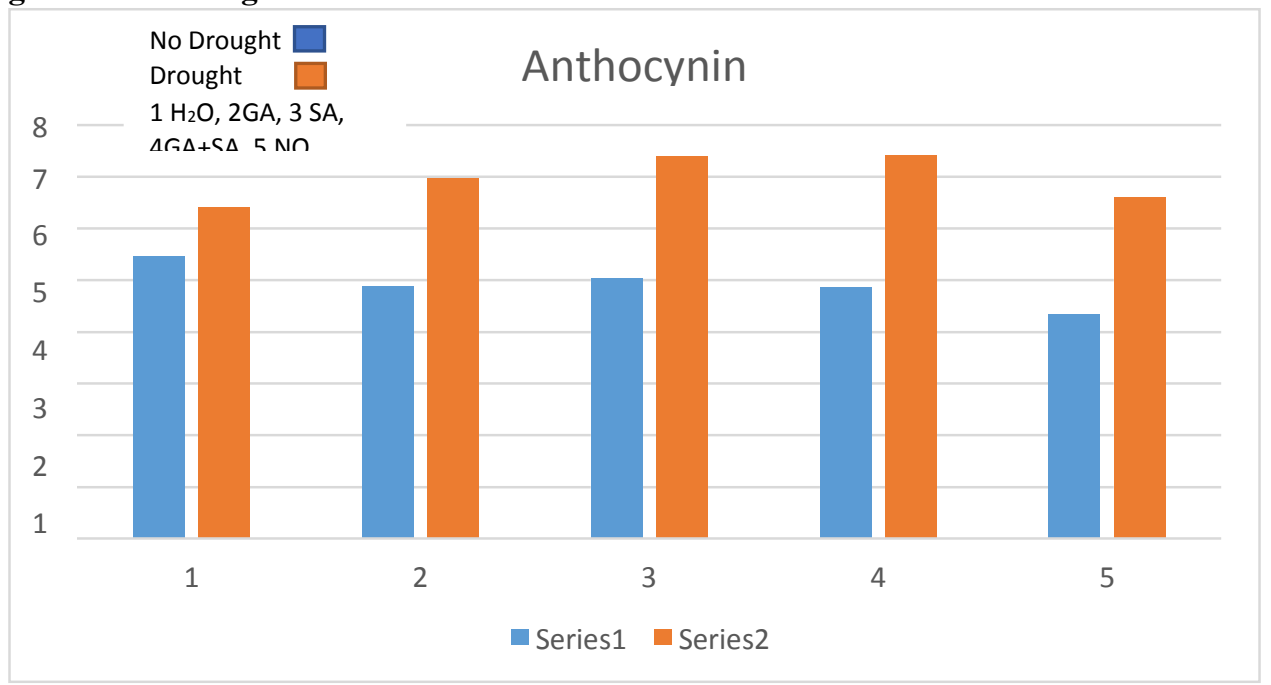


Figure-5 Effect of different hormones on sugar content $\mathrm{mg} / \mathrm{g}$ fresh weight of drought stressed maize plant. No Drought $\square$

Drought Sugar

$2-1 \mathrm{H} 2 \mathrm{O}, 2 \mathrm{GA}, 3 \mathrm{SA}, 4 \mathrm{GA}+\mathrm{SA}$,

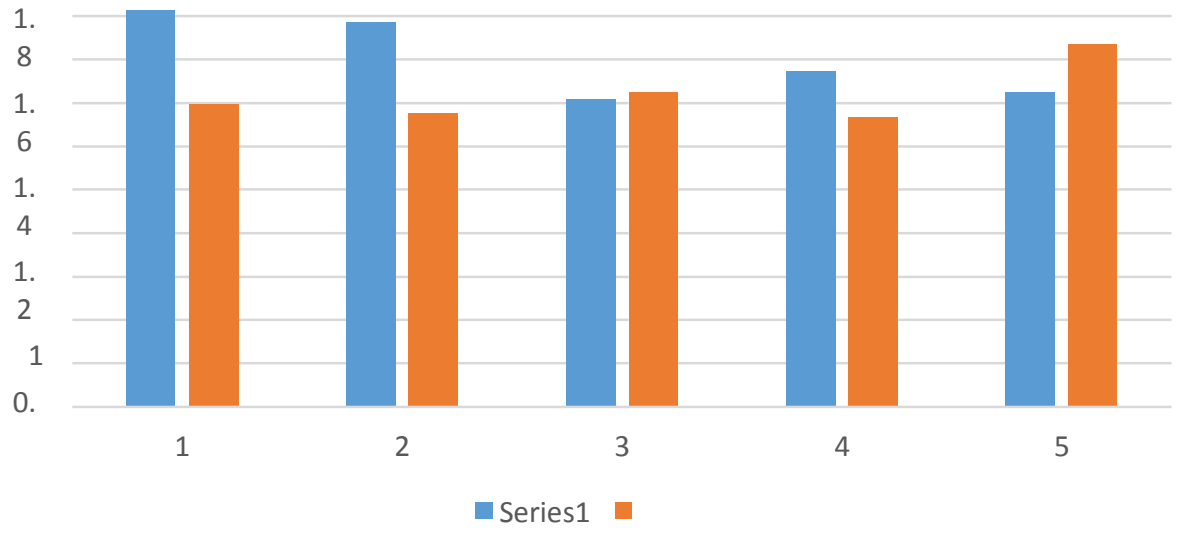

Figure-6 Effect of different hormones on proline content (mg/g fresh weight) under drought stressed maize plant.

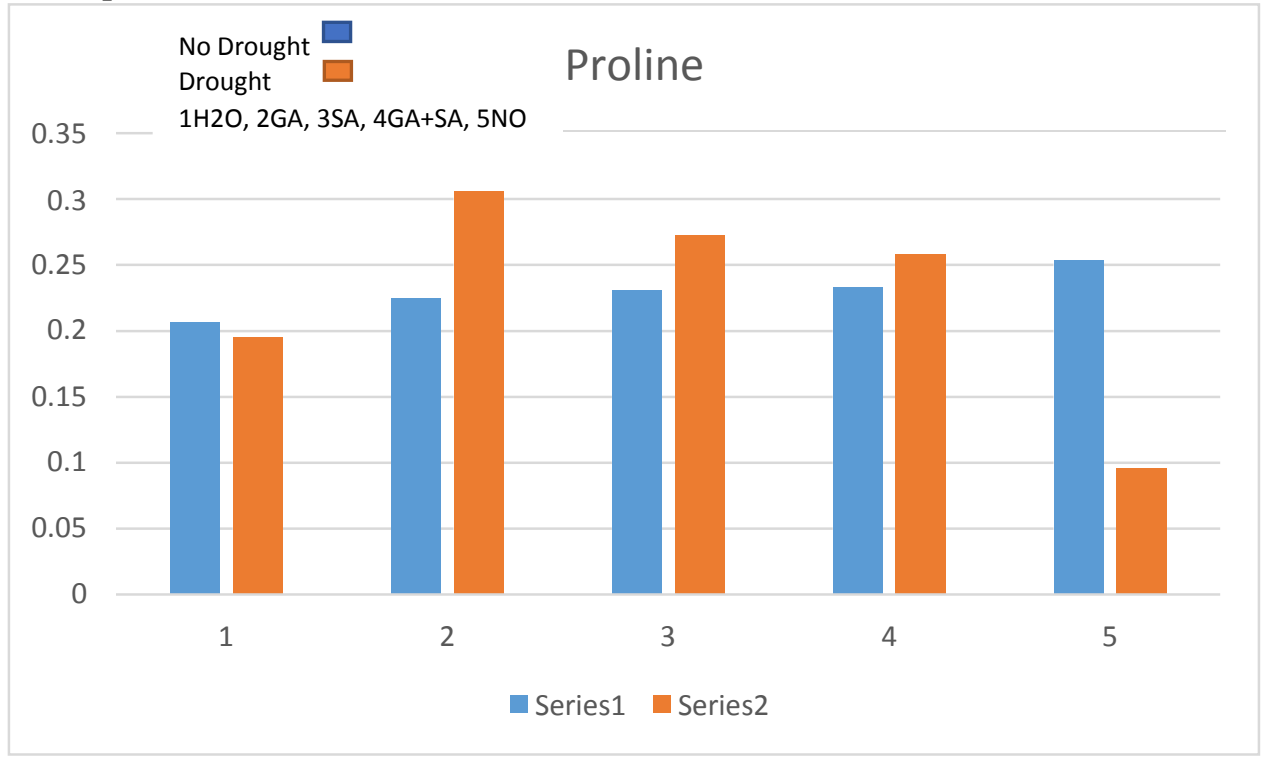


Figure-7 Effect of different hormones on protein content $(\mathrm{mg} / \mathrm{g})$ of drought stress maize plant at post germination stage.

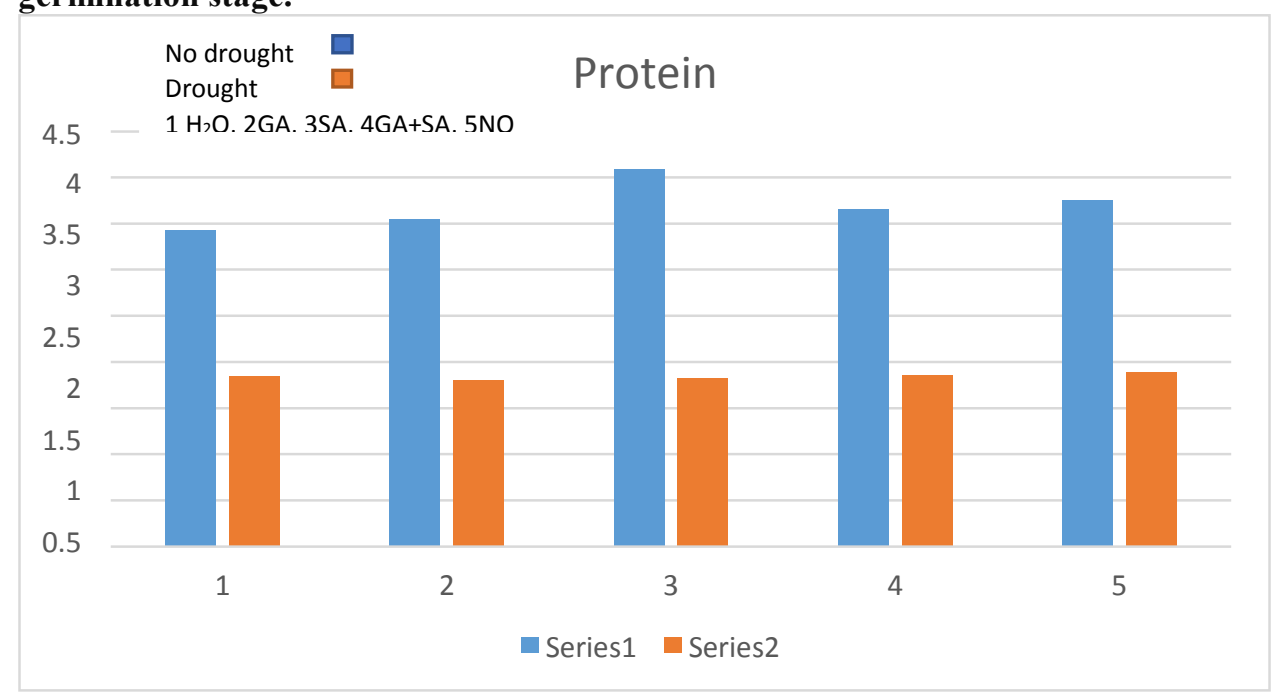

Figure-8 Effect of different hormones on catalase activity ( $\mu \mathrm{mol}$ hydrogen peroxide reduced $/ \mathrm{min} / \mathrm{mg}$ protein) of drought stress maize plant.

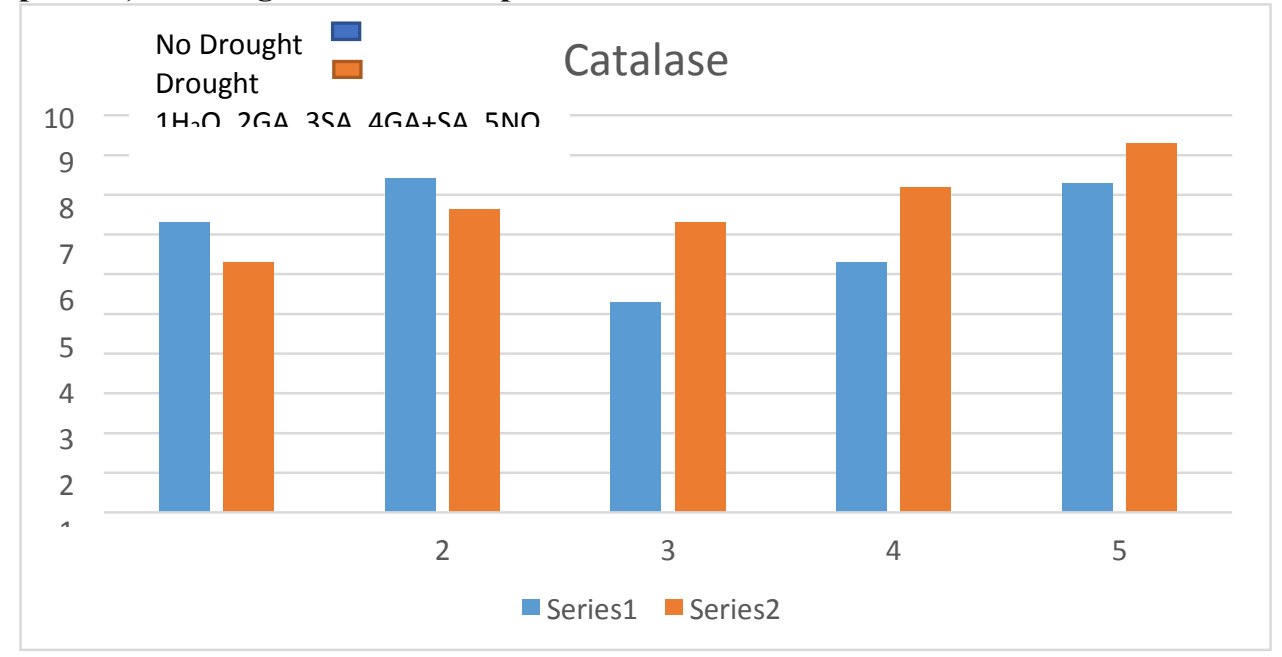


Figure-9 Effect of different hormones on peroxidase activity (EU/min/ mg) of maize plant under drought stress.

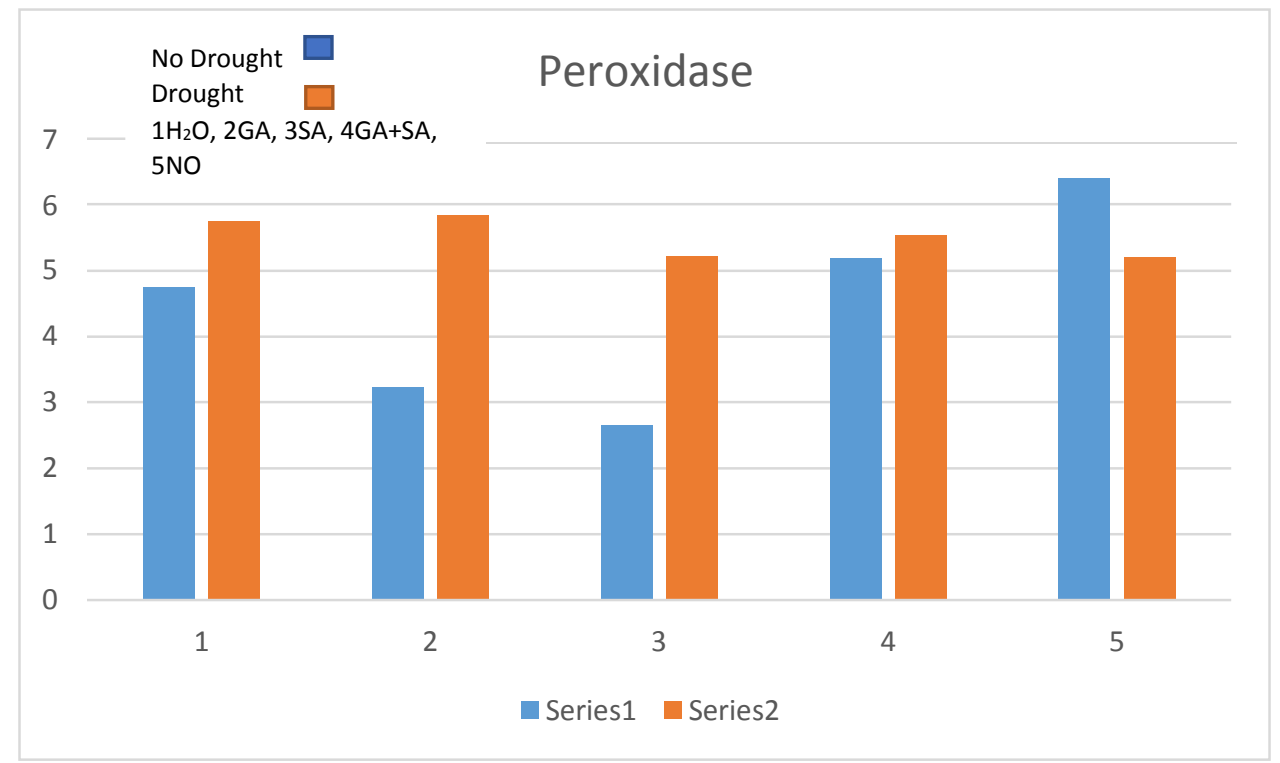

Figure-10: Effects of different hormones on ascorbate-peroxidase activity ( $\mu \mathrm{mol}$ ascorbate oxidize $/ \mathrm{min} / \mathrm{mg}$ protein) of drought stressed maize plant.

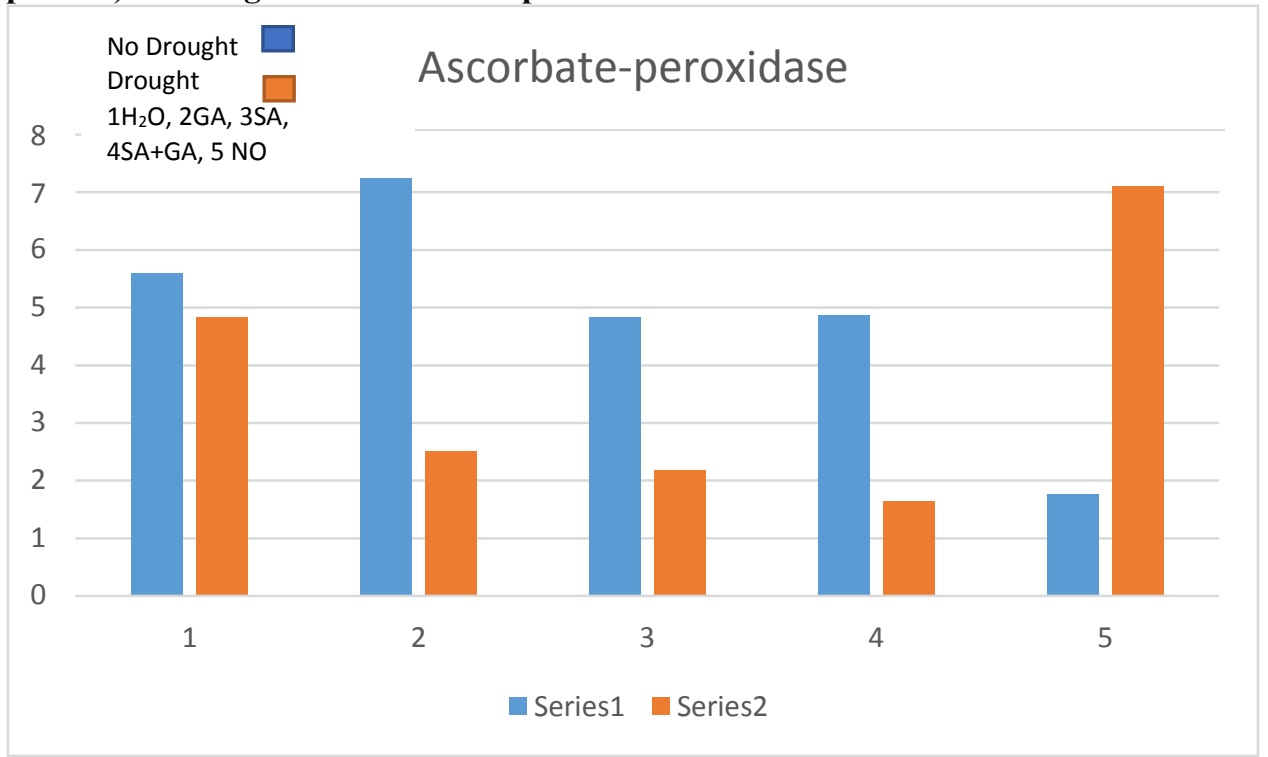


Figure-11 Effect of different hormones on polyphenol oxidase activity (EU/min/mg protein) under drought stressed maize plant at post germination stage.

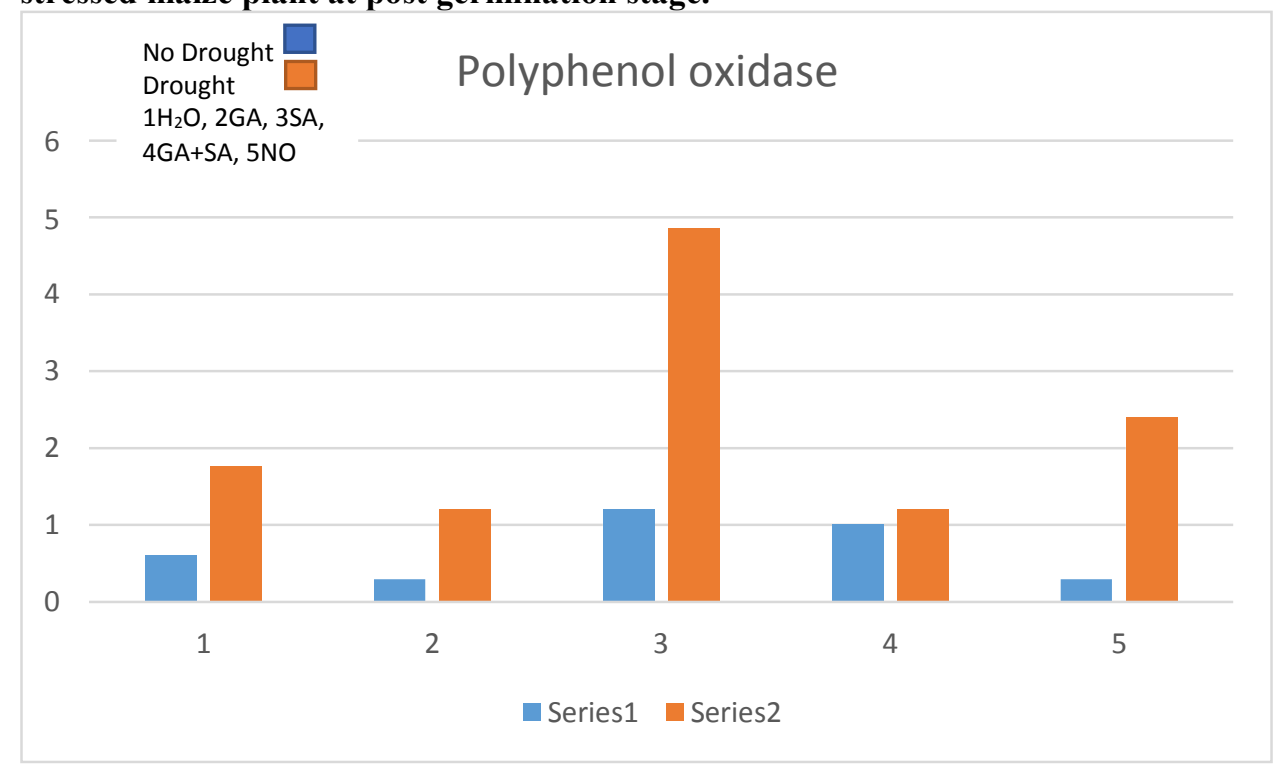

Figure-12: Effect of different growth hormones on total chlorophyll content $(\mathrm{mg} / \mathrm{g}$ fw) of post germination drought stress maize.

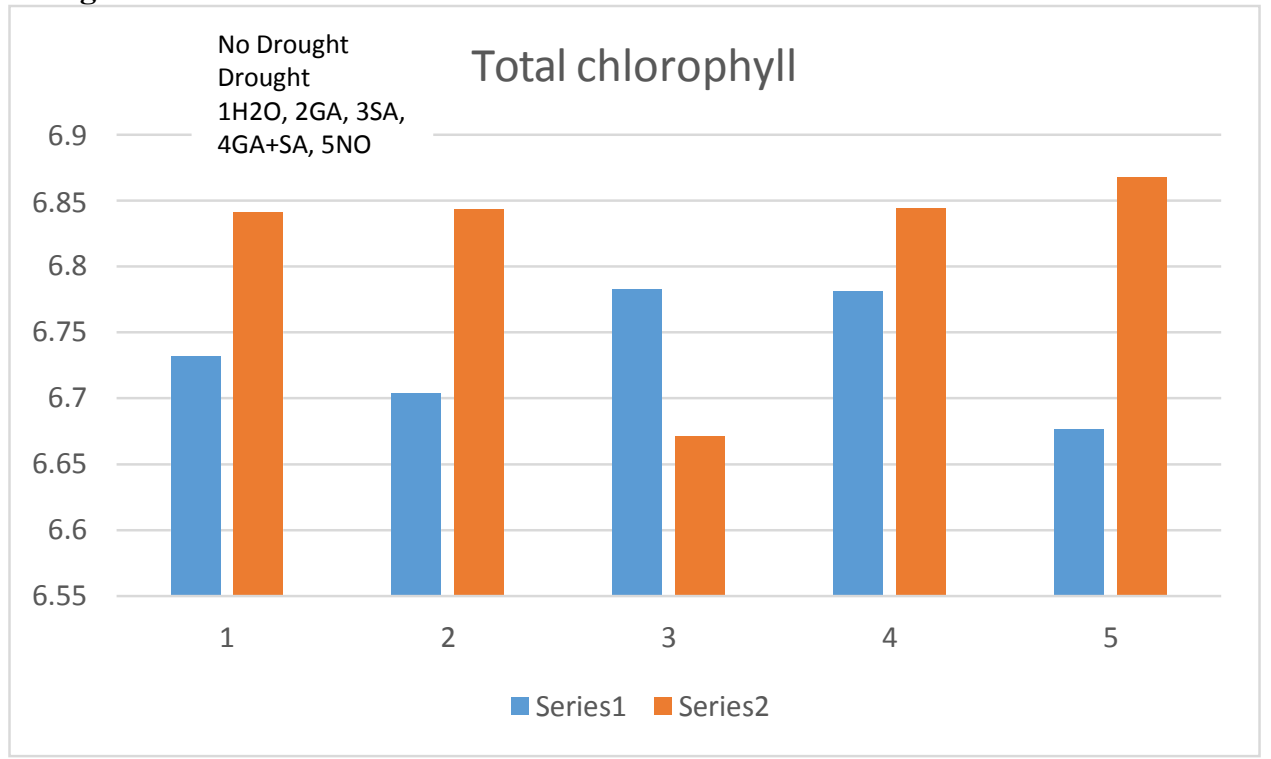


Figure-13: Effect of different growth hormones on chlorophyll $a / b(\mathrm{mg} / \mathrm{g} \mathrm{fw})$ of post germination drought stress maize.

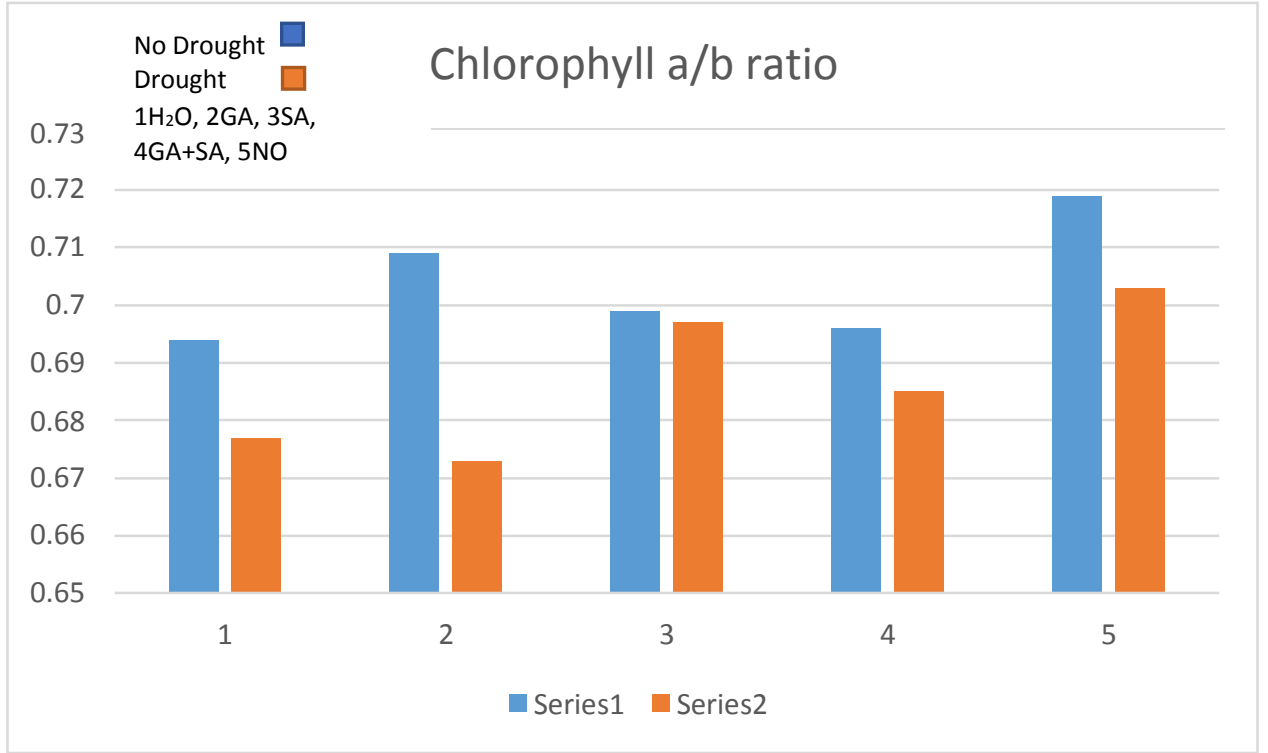

